\title{
Comparison of molecular profiles of human mesenchymal stem cells derived from bone marrow, umbilical cord blood, placenta and adipose tissue
}

\author{
JUNE SEOK HEO $^{1}$, YOUJEONG CHOI ${ }^{1}$, HAN-SOO KIM ${ }^{3,4}$ and HYUN OK KIM ${ }^{1,2}$ \\ ${ }^{1}$ Cell Therapy Center, Severance Hospital; ${ }^{2}$ Department of Laboratory Medicine, Yonsei University \\ College of Medicine, Seoul; ${ }^{3}$ Institute for Bio-Medical Convergence, Catholic Kwandong University, Incheon; \\ ${ }^{4}$ Department of Biomedical Sciences, Catholic Kwandong University, Gangneung, Republic of Korea
}

Received June 19, 2015; Accepted November 17, 2015

DOI: $10.3892 /$ ijmm.2015.2413

\begin{abstract}
Mesenchymal stem cells (MSCs) are clinically useful due to their capacity for self-renewal, their immunomodulatory properties and tissue regenerative potential. These cells can be isolated from various tissues and exhibit different potential for clinical applications according to their origin, and thus comparative studies on MSCs from different tissues are essential. In this study, we investigated the immunophenotype, proliferative potential, multilineage differentiation and immunomodulatory capacity of MSCs derived from different tissue sources, namely bone marrow, adipose tissue, the placenta and umbilical cord blood. The gene expression profiles of stemness-related genes [octamerbinding transcription factor 4 (OCT4), sex determining region Y-box (SOX)2, MYC, Krüppel-like factor 4 (KLF4), NANOG, LIN28 and REXI] and lineage-related and differentiation stage-related genes [B4GALNT1 (GM2/GS2 synthase), inhibin, beta A (INHBA), distal-less homeobox 5 (DLX5), runt-related transcription factor $2(R U N X 2)$, proliferator-activated receptor gamma $(P P A R G)$, CCAAT/enhancer-binding protein alpha $(C / E B P A)$, bone morphogenetic protein 7 (BMP7) and $S O X 9$ ] were compared using RT-PCR. No significant differences in growth rate, colony-forming efficiency and immunophenotype were observed. Our results demonstrated that MSCs derived from bone marrow and adipose tissue shared not only in vitro tri-lineage differentiation potential, but also gene expression profiles. While there was considerable inter-
\end{abstract}

Correspondence to: Dr Hyun Ok Kim, Department of Laboratory Medicine, Yonsei University College of Medicine, 250 Seongsanno, Seodaemun-gu, Seoul 120-752, Republic of Korea

E-mail: hyunok1019@yuhs.ac

Dr Han-Soo Kim, Department of Biomedical Sciences, Catholic Kwandong University, 24 Beomil-ro 579beon-gil, Gangneung-si, Gangwon-do 210-701, Republic of Korea

E-mail: hankim63@gmail.com

Key words: distal-less homeobox 5, mesenchymal stem cells, multipotency donor variation in DLX5 expression between MSCs derived from different tissues, its expression appears to be associated with the osteogenic potential of MSCs. Bone marrow-derived MSCs (BM-MSCs) significantly inhibited allogeneic T cell proliferation possibly via the high levels of the immunosuppressive cytokines, ILIO and TGFB1. Although MSCs derived from different tissues and fibroblasts share many characteristics, some of the marker genes, such as B4GALNT1 and DLX5 may be useful for the characterization of MSCs derived from different tissue sources. Collectively, our results suggest that, based on their tri-lineage differentiation potential and immunomodulatory effects, BM-MSCs and adipose tissue-derived MSCs (A-MSCs) represent the optimal stem cell source for tissue engineering and regenerative medicine.

\section{Introduction}

Mesenchymal stem cells (MSCs) are multipotent somatic stem cells that have the potential to differentiate into mesodermal and even non-mesodermal lineages and are known to produce trophic factor for tissue repair/regeneration (1-4). Due to their ease of isolation, culture expansion, multipotential differentiation and immunomodulatory properties, MSCs have the potential for use in regenerative medicine and have therapeutic application (5). Indeed, MSCs have been investigated in a number of clinical trials for presently untreatable diseases, such as bone and cartilage defects, myocardial infarction, stroke, graft-versus-host disease (GvHD) and autoimmune diseases $(6,7)$.

Although MSCs were first reported to be derived from bone marrow, a number of studies have reported similar cell types in a wide range of tissues, e.g., umbilical cord blood, the placenta, adipose tissue, amniotic fluid, dental tissue, skin, hair follicles and tonsils (8-14). Given the observed clinical efficacy of MSCs and a number of comparative analyses of MSCs derived from different tissues, it is surprising that so little is known about the identity and characteristics of MSCs derived from different tissues $(15,16)$. Although the International Society for Cellular Therapy (ISCT) proposed the minimal criteria of MSCs in 2006, this definition is non-specific and fails to address the differences between MSCs (derived from different tissues) and fibroblasts (17). 
Currently, there is no consensus on the markers that identify or distinguish MSCs derived from different tissues and fibroblasts. Furthermore, a precise characterization of MSCs derived from different tissues and their properties relating to their therapeutic potential represent an essential requirement for the exploitation and development of optimal MSC-based therapies, since the biological capacity of MSCs (i.e., immunomodulatory capacity, differentiation potential to a specific cell type and endogenous stem cell mobilizing capacity) of one tissue may be superior to others.

The aim of the present study was to compare the biological characteristics of MSCs originating from different tissues, i.e., bone marrow (BM-MSCs), umbilical cord blood (CB-MSCs), placenta (P-MSCs) and adipose tissue (A-MSCs), with respect to cell morphology, growth rate, immunophenotype, gene expression profile, immunomodulatory capacity and differentiation potential under the same conditions. The characterization of MSCs derived from different tissues with identifying molecular signatures may prove to be helpful for selecting a suitable source for a specified clinical application.

\section{Materials and methods}

Cells. Bone marrow samples (from 3 male donors, aged 21, 26 and 27 years, respectively) were obtained from normal allogeneic hematopoietic stem cell donors after obtaining written informed consent. Umbilical cord blood was collected in a bag with CPDA anticoagulant following delivery (from 3 donor babies, 1 male and 2 females). This study was approved by the Institutional Review Boards of Severance Hospital (an affiliated hospital of Yonsei University Health System, Seoul, Korea). The mononuclear cell (MNC) fraction was separated by FicollHypaque density gradient centrifugation (Pharmacia Biotech, Uppsala, Sweden), and the MSCs were cultured as previously described (18). Human dermal fibroblasts (from 3 donors, a 22 year-old female, 26 year-old female and 31 year-old male) were provided by Dr Dong-Wook Kim (Yonsei University College of Medicine, Korea). In this study, fibroblasts were used as a negative control. Placental- (from 3 donors, 28-, 32- and 33-year-old females) and adipose tissue-derived MSCs (from 3 donors, 34-, 41- and 46-year old females) were kindly provided by Dr Ja Young Kwon (Yonsei University College of Medicine) and Dr Kyoung Sik Kim (Yonsei University College of Medicine), respectively. The isolated MSCs were frozen until the cells were used. To permit an exact analysis, all cells were used at passage 3-5 and cultured under standardized conditions; DMEM-low glucose supplemented with $10 \%$ fetal bovine serum (FBS) and 1\% penicillin/streptomycin (P/S) (all from Invitrogen, Carlsbad, CA, USA). The cells were cultured at $37^{\circ} \mathrm{C}$ with $5 \% \mathrm{CO}_{2}$, and the media were replaced every 3 or 4 days. Over the course of expansion, we examined the differences in cell morphology under an inverted phase microscope (Olympus IX-71; Olympus, Tokyo, Japan).

Growth characteristics. To compare the growth characteristics of the cells, the growth rate and population doubling time (PDT; period of time required for cells to proliferate or grow) were measured. All cells were plated at a density of $2 \times 10^{4}$ cells in 12-well plates. On days 2 and 4, the cells were harvested and counted by Trypan blue staining. The PDT was calculated based on a previously reported formula (19). The finite population doublings were defined as the cumulative number of serial cell passages until the cells reached senescence.

Colony-forming unit-fibroblast (CFU-F) assay. The capacity of the cells for self-renewal can be evaluated by CFU-F assay. To assess the self-renewal capacity of the cells, $1 \times 10^{3}$ cells at passage 3 were seeded in 100-mm plates (Corning Inc., Corning, NY, USA). Following cultivation for 14 days, the cells were washed with phosphate-buffered saline (PBS; Invitrogen) and stained with $0.5 \%$ crystal violet (Sigma-Aldrich, St. Louis, MO, USA) for $5 \mathrm{~min}$ at room temperature. Stained colonies with $>50$ cells were counted.

Immunophenotyping. The cells were stained with the following antibodies: CD14-FITC (555397), CD29-FITC (556048), CD31-PE (555446), CD34-FITC (560942), CD44-PE (555479), CD45-PE (561866), CD73-PE (550257), CD90-FITC (555595), CD105-PE (560839) and CD106-FITC (551146) (all from BD Pharmingen, San Diego, CA, USA). Additionally, phycoerythrin-conjugated and FITC-conjugated isotype controls were applied. The cells were stained with the antibodies for 20 min at $4^{\circ} \mathrm{C}$. The stained cells were washed with PBS and fixed with $1 \%$ paraformaldehyde (Biosesang, Seongnam, Korea). Subsequently, the labeled cells were analyzed using a flow cytometer (Cytomics Flow Cytometer; Beckman Coulter, Fullerton, CA, USA).

$R N A$ isolation and RT-PCR. Total RNA was extracted using TRIzol reagent (Invitrogen). Standard reverse transcription (RT) was performed using transcriptase II (Invitrogen). RT-PCR was performed using PCR primers (Bioneer, Daejeon, Korea) under the conditions listed in Table I. The glyceraldehyde 3-phosphate dehydrogenase (GAPDH) level was used as an internal control. Human induced pluripotent stem (hiPS) cell cDNA was used as a positive control (kindly provided by Dr Dong-Wook Kim, Yonsei University College of Medicine). The signal intensity of the product was normalized to its respective GAPDH signal intensity.

Differentiation assay. To induce osteogenic, adipogenic and chondrogenic differentiation, the cells derived from each type of tissue were seeded simultaneously in osteogenic induction medium, chondrogenic induction medium, and adipogenic induction medium (Cambrex, Lonza, MD, USA). The cells were then cultured for 3 weeks, and the medium was changed every 3 or 4 days. Whenever the medium was changed during chondrogenesis, $10 \mathrm{ng} / \mathrm{ml}$ transforming growth factor (TGF)- $\beta 3$ (Cambrex) was added. After 3 weeks, the cells were analyzed for osteogenesis, adipogenesis and chondrogenesis by von Kossa staining, Oil Red O staining, and Safranin O staining. The stained cells were photographed using a phase microscope (Olympus IX-71; Olympus).

$T$ cell proliferation assay. To assess the ability of MSCs to suppress T cell proliferation, the MSCs were treated with $50 \mathrm{ng} / \mathrm{ml}$ of mitomycin C (Sigma-Aldrich) for $60 \mathrm{~min}$ to inactivate their proliferation. Subsequently, $2 \times 10^{5}$ cells of human peripheral blood MNCs were co-cultured with $2 \times 10^{4}$ MSCs of each type in a 96-well plate. To activate T cells, $10 \mu \mathrm{g} / \mathrm{ml}$ 
Table I. Primer sets used for RT-PCR.

\begin{tabular}{|c|c|c|c|}
\hline Gene & Primer sequence $\left(5^{\prime} \rightarrow 3^{\prime}\right)$ & Annealing temperature $\left({ }^{\circ} \mathrm{C}\right)$ & Product size (bp) \\
\hline \multirow[t]{2}{*}{ GAPDH } & Forward: GTGGTCTCCTCTGACTTCAACA & & \\
\hline & Reverse: CTCTTCCTCTTGTGCTCTTGCT & 62 & 210 \\
\hline \multirow[t]{2}{*}{ OCT4 } & Forward: GACAACAATGAGAACCTTCAGGAGA & & \\
\hline & Reverse: TTCTGGCGCCGGTTACAGAACCA & 62 & 218 \\
\hline \multirow[t]{2}{*}{ SOX2 } & Forward: AACCAAGACGCTCATGAAGAAG & & \\
\hline & Reverse: GCGAGTAGGACATGCTGTAGGT & 62 & 341 \\
\hline \multirow[t]{2}{*}{ c-Myc } & Forward: TCGGATTCTCTGCTCTCCTC & & \\
\hline & Reverse: CGCCTCTTGACATTCTCCTC & 62 & 413 \\
\hline \multirow[t]{2}{*}{ KLF4 } & Forward: ATTCTCTCCAATTCGCTGACCC & & \\
\hline & Reverse: TTCAGCACGAACTTGCCCAT & 62 & 376 \\
\hline \multirow[t]{2}{*}{ NANOG } & Forward: ATAGCAATGGTGTGACGCAG & & \\
\hline & Reverse: GATTGTTCCAGGATTGGGTG & 62 & 219 \\
\hline \multirow[t]{2}{*}{ REX1 } & Forward: CTGAAGAAACGGGCAAAGAC & & \\
\hline & Reverse: GAACATTCAAGGGAGCTTGC & 58 & 344 \\
\hline \multirow[t]{2}{*}{ LIN28 } & Forward: GCTCCGTGTCCAACCAGCAG & & \\
\hline & Reverse: TTTCCTTTTGGCCGCCTCTC & 58 & 376 \\
\hline \multirow[t]{2}{*}{ GD2 synthase } & Forward: CCAACTCAACAGGCAACTAC & & \\
\hline & Reverse: GATCATAACGGAGGAAGGTC & 59 & 230 \\
\hline \multirow[t]{2}{*}{ DLX5 } & Forward: ACCATCCGTCTCAGGAATCG & & \\
\hline & Reverse: ACCTTCTCTGTAATGCGGCC & 60 & 384 \\
\hline \multirow[t]{2}{*}{ CBFA1 } & Forward: TTGCAGCCATAAGAGGGTAG & & \\
\hline & Reverse: GTCACTTTCTTGGAGCAGGA & 58 & 470 \\
\hline \multirow[t]{2}{*}{ PPARG } & Forward: TCTCTCCGTAATGGAAGACC & & \\
\hline & Reverse: GCATTATGAGACATCCCCAC & 55 & 474 \\
\hline \multirow[t]{2}{*}{ C/EBPA } & Forward: CCAAGAAGTCGGTGGACAAGAA & & \\
\hline & Reverse: TCATTGTCACTGGTCAGCTCCA & 62 & 145 \\
\hline \multirow[t]{2}{*}{ BMP7 } & Forward: CCAACGTCATCCTGAAGAAATAC & & \\
\hline & Reverse: GCTTGTAGGATCTTGTTCATTGG & 60 & 271 \\
\hline \multirow[t]{2}{*}{ SOX9 } & Forward: GGTTGTTGGAGCTTTCCTCA & & \\
\hline & Reverse: TAGCCTCCCTCACTCCAAGA & 61 & 400 \\
\hline \multirow[t]{2}{*}{ HLA-ABC } & Forward: CAGATACCTGGAGAACGG & & \\
\hline & Reverse: TGGCCTCATGGTCAGAGA & 56 & 96 \\
\hline \multirow[t]{2}{*}{ HLA-DR } & Forward: CCCCACAGCACGTTTCTTG & & \\
\hline & Reverse: CCGCTGCACTGTGAAGCTCT & 60 & 274 \\
\hline \multirow[t]{2}{*}{ HLA-G } & Forward: GCGGCTACTACAACCAGAGC & & \\
\hline & Reverse: GCACATGGCACGTGTATCTC & 58 & 900 \\
\hline \multirow[t]{2}{*}{ IL10 } & Forward: ACCTGGTAGAAGTGATGCCCCAGGCA & & \\
\hline & Reverse: CTATGCAGTTGATGAAGATGTCAA & 58 & 237 \\
\hline \multirow[t]{2}{*}{ TNFAIP6 } & Forward: GGTGTGTACCACAGAGAAGCA & & \\
\hline & Reverse: GGGTTGTAGCAATAGGCATCC & 60 & 284 \\
\hline \multirow[t]{2}{*}{ TSG-6 } & Forward: GGTGTGTACCACAGAGAAGCA & & \\
\hline & Reverse: GGGTTGTAGCAATAGGCATCC & 60 & 284 \\
\hline \multirow[t]{2}{*}{ IL6 } & Forward: ATGAACTCCTTCTCCACAAGC & & \\
\hline & Reverse: GTTTCTGCCAGTGCCTCTTTG & 60 & 264 \\
\hline \multirow[t]{2}{*}{ TGFB1 } & Forward: GAGGTGACCTGGCCACCATT & & \\
\hline & Reverse: TCCGCAAGGACCTCGGCTGG & 55 & 194 \\
\hline INHBA & Forward: GATGTACCCAACTCTCAGCCA & & \\
\hline & Reverse: GCCGATGTCCTTGAAACTGAC & 55 & 866 \\
\hline
\end{tabular}

SOX2, sex determining region Y-box 2; DLX5, distal-less homeobox 5; C/EBPA, CCAAT/enhancer-binding protein alpha; BMP7, bone morphogenetic protein 7; IL, interleukin ; TGFB1, transforming growth factor beta 1. OCT4, octamer-binding transcription factor 4; KLF4, Krüppel-like factor 4; DLX5, distal-less homeobox 5; CBFA1, core-binding factor alpha (1); PPARG, proliferator-activated receptor gamma; TNFAIP6, tumor necrosis factor, alpha-induced protein 6; TSG-6, tumor necrosis factor-stimulated gene 6 (TSG-6); IHHBA, Activin A, inhibin beta A. 

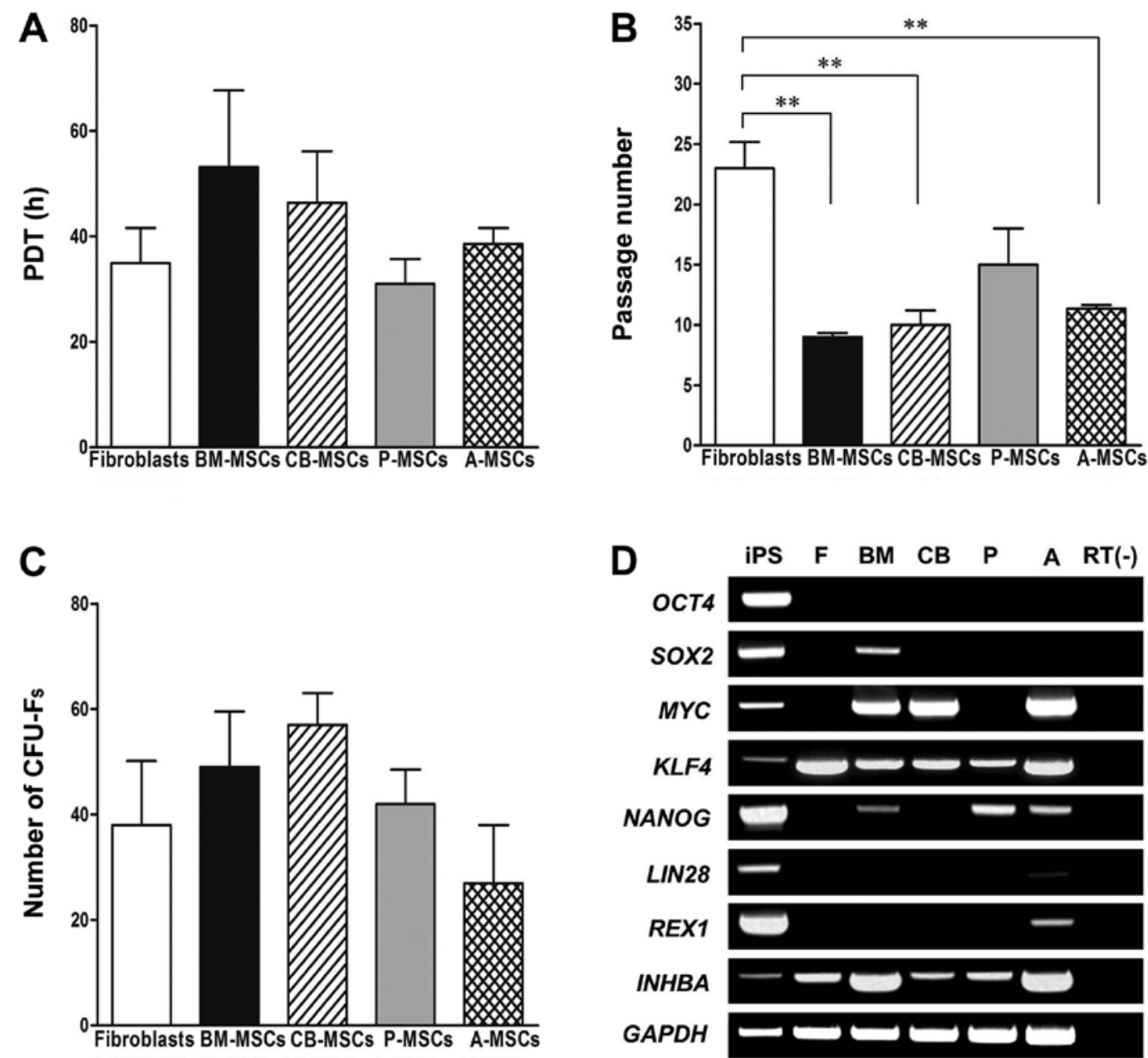

Figure 1. Growth characteristics and stemness marker expression of human mesenchymal stem cells (MSCs) derived from different tissues. (A) Growth kinetics. Population doubling time (PDT, measured in hours) was determined at each subcultivation. MSCs derived from bone marrow (BM-MSCs), umbilical cord blood (CB-MSCs), the placenta (P-MSCs) and adipose tissue (A-MSCs) that were cultured under identical conditions. (B) Long-term expandability. The finite population doublings, defined as the total number of serial cell passaging before reaching replicative senescence. ${ }^{* *}$ p $<0.01$. (C) Clonogenic capacity was measured by colony forming unit-fibroblast (CFU-F) assay. The results (A-C) were obtained from 3 independent donors and are represented as the means \pm SD. (D) Stemness marker expression in MSCs derived from different tissues. RT-PCR analysis for pluripotency markers in MSCs derived from bone marrow, umbilical cord blood, the placenta and adipose tissue compared to induced pluripotent stem (iPS) cell and fibroblasts (F). RT(-) denotes the absence of reverse transcriptase as a control. One representative of 3 independent experiments is shown.

phytohaemagglutinin (PHA; Sigma-Aldrich) was applied for $72 \mathrm{~h}$. To examine the inhibition of T cells, a BrdU cell proliferation assay (Millipore, Billerica, MA, USA) was performed according to the manufacturer's instructions. Activated T cells alone without MSCs were used as a positive control.

Statistical analysis. Quantitative data are expressed as the means \pm SD. All statistical comparisons between groups were performed by one-way analysis of variance (ANOVA) with post hoc Bonferroni corrections. A p-value $<0.05$ was considered to indicate a statistically significant difference.

\section{Results}

Growth characteristics of MSCs derived from different tissues. All MSCs and fibroblasts exhibited similar growth properties on day 2. However, of the MSCs derived from different tissues, the P-MSCs displayed the highest proliferative capacity between days 2 and 4 (Fig. 1A), as they had the lowest PDT.
Although the P-MSCs showed a slight increase in growth compared to the controls (shown by the decrease in the PDT), the differences in population doubling time between the tested cells were not statistically significant. Although the cells were isolated from different tissues, we did not find any differences in our morphological examination (data not shown). To determine the maximum proliferative capacity, all cell types were serially passaged until they displayed replicative senescence with a loss of proliferation. Of the MSCs derived from different tissues, the P-MSCs could withstand longer periods of culture, whereas the BM-, CB- and A-MSCs exhibited a similar maximum culture period (Fig. 1B). The CFU-F assay was used to examine the self-renewal capacity of the cells. Although the fibroblasts and P-MSCs exhibited better growth characteristics than the other cells, there were no significant differences in the number of CFU-Fs following cell seeding at $1 \times 10^{3}$ cells in 100-mm plates after 14 days (Fig. 1C). The BM- and CB-MSCs displayed a higher self-renewal capacity regardless of growth rate, although the differences were not significant. 
Table II. Immunophenotyping of cells derived from various sources by flow cytometry.

\begin{tabular}{|c|c|c|c|c|c|}
\hline Surface marker & Fibroblasts & BM-MSCs & CB-MSCs & P-MSCs & A-MSCs \\
\hline CD14 & $1.0 \pm 1.5^{\mathrm{a}}$ & $1.4 \pm 0.8$ & $1.3 \pm 0.6$ & $0.8 \pm 0.8$ & $2.0 \pm 1.1$ \\
\hline CD29 & $82.3 \pm 15.6$ & $97.5 \pm 1.9$ & $94.4 \pm 7.9$ & $98.6 \pm 1.9$ & $68.5 \pm 17.9$ \\
\hline CD31 & $1.2 \pm 0.4$ & $1.8 \pm 1.4$ & $1.0 \pm 0.9$ & $0.4 \pm 0.5$ & $0.6 \pm 0.5$ \\
\hline CD34 & $0.8 \pm 1.2$ & $2.3 \pm 1.6$ & $6.1 \pm 8.4$ & $0.7 \pm 0.7$ & $1.8 \pm 1.1$ \\
\hline CD44 & $93.3 \pm 11.7$ & $100 \pm 0.0$ & $96.5 \pm 6.0$ & $93.0 \pm 12.0$ & $99.8 \pm 0.3$ \\
\hline CD45 & $0.7 \pm 0.6$ & $1.7 \pm 1.5$ & $0.5 \pm 0.4$ & $0.4 \pm 0.3$ & $0.9 \pm 0.4$ \\
\hline CD73 & $99.3 \pm 0.3$ & $99.1 \pm 0.9$ & $93.7 \pm 6.3$ & $99.5 \pm 0.8$ & $90.9 \pm 3.0$ \\
\hline CD90 & $90.9 \pm 10.1$ & $83.1 \pm 20.3$ & $66.4 \pm 11.2$ & $22.2 \pm 12.7$ & $62.5 \pm 16.9$ \\
\hline CD105 & $91.7 \pm 0.6$ & $90.3 \pm 12.1$ & $68.2 \pm 27.2$ & $73.5 \pm 36.3$ & $75.0 \pm 14.1$ \\
\hline CD106 & $1.0 \pm 1.6$ & $4.3 \pm 1.6$ & $6.3 \pm 7.5$ & $0.9 \pm 1.1$ & $2.0 \pm 1.2$ \\
\hline
\end{tabular}

${ }^{a}$ Means \pm SD of 3 experiments. BM-MSCs, bone marrow-derived mesenchymal stem cells; CB-MSCs, umbilical cord blood-derived mesenchymal stem cells; P-MSCs, placenta-derived mesenchymal stem cells; A-MSCs, adipose tissue-derived mesenchymal stem cells.

In order to identify the molecular signature, we examined the expression of stemness markers in the MSCs derived from different tissues (Fig. 1D). The octamer-binding transcription factor 4 (OCT4) gene was not detected in any of the MSCs, or the fibroblasts. Sex determining region Y-box 2 (SOX2) was only expressed in the BM-MSCs; NANOG was detected in the BM-, P- and A-MSCs. Compared to the hiPS cells, the expression of SOX2 and NANOG was much lower in the BM-MSCs. Krüppel-like factor 4 (KLF4) was expressed in all types of cells and fibroblasts, whereas $M Y C$ was expressed in all cells apart from the fibroblasts and P-MSCs. Activin A [inhibin, beta A (INHBA)] was strongly detected in the BMand A-MSCs, as compared to the fibroblasts and MSCs derived from other tissues. Compared to the hiPS cells, MYC, KLF4 and INHBA expression was much stronger in the other MSCs tested. In the A-MSCs we noted a basal expression of LIN28 and $R E X 1$, which was much lower than that expressed in the hiPS cells. These results suggest that BM- and A-MSCs possess the highest capacity for self-renewal and differentiation potential in multiple lineages, whereas P-MSCs have the least functionality as stem cells of those which were tested.

Immunophenotype and differentiation potential. Flow cytometric analysis was performed with the MSCs derived from different tissues, and we revealed that all cell types displayed similar immunophenotypic patterns. The cells were negative for CD14, CD31, CD34, CD45 and CD106, which are known markers of hematopoietic and endothelial cells, whereas the MSCs were positive for CD29, CD44, CD73, CD90 and CD105, which are known markers of MSCs. Positive MSC markers were expressed in all of the cell types, even in fibroblasts (Table II). These results confirm that cells from diverse sources express MSC surface markers, as defined by the ISCT. However, the expression of CD90, a typical MSC marker, was less obvious in the P-MSCs than in the other cells.

To investigate the differentiation potential of the MSCs, the cells were subjected to osteogenic, adipogenic and chondrogenic differentiation (Fig. 2A). Osteogenic differentiation, which was evaluated by calcium deposition and von Kossa staining, was evident in the BM- and A-MSCs, whereas the other MSCs did not differentiate into osteoblasts under osteogenic induction. No osteogenic differentiation was induced in the fibroblasts. Adipogenic differentiation, verified by the accumulation of cytoplasmic lipid vacuoles and Oil Red O staining, was distinctly observed in the BM- and A-MSCs, whereas theCB- and P-MSCs were only weakly positive. Only a few or very small Oil Red O-stained granules were detected in the fibroblasts, and this could be explained by the findings of a previous study which suggested that human dermal fibroblasts exhibit delayed adipogenic differentiation compared with MSCs (as also shown in Fig. 3A) (20). Chondrogenesis, verified by cartilage-specific proteoglycans and Safranin O staining, was demonstrated in all the tested cells (Fig. 3B). The BM- and A-MSCs exhibited only tri-lineage potency, whereas the CB- and P-MSCs had the capacity to differentiate into only 2 cell lineages. The fibroblasts also differentiated into adipocytes and chondrocytes, although the results were weakly positive. Therefore, we suggest that only the BM- and A-MSCs can differentiate into 3 mesodermal lineages, i.e., osteoblasts, adipocytes and chondrocytes, thus demonstrating that of the cells from diverse sources, only the BM- and A-MSCs have multipotency as true MSCs.

Subsequently, we evaluated the osteogenic, adipogenic and chondrogenic gene expression in the cells by RT-PCR (Fig. 2B). Osteogenesis-related gene runt-related transcription factor 2 ( $R U N X 2)$, adipogenesis-related genes peroxisome proliferator-activated receptor gamma (PPARG), CCAAT/ enhancer-binding protein alpha $(C / E B P A)$, and chondrogenesis-related genes bone morphogenetic protein 7 (BMP7) and sex determining region Y-box 9 (SOX9) were similarly expressed in the majority of cell types, whereas distal-less homeobox 5 (DLX5), which plays a key role in the development of skeletal elements and the commitment of MSCs to the osteoblast lineage was only expressed in the BM-MSCs and A-MSCs. RUNX2 and PPARG expression in the BM-MSCs were lower than in the other cell types. These results again support our theory that BM- and A-MSCs possess tri-lineage differentiation potential. 


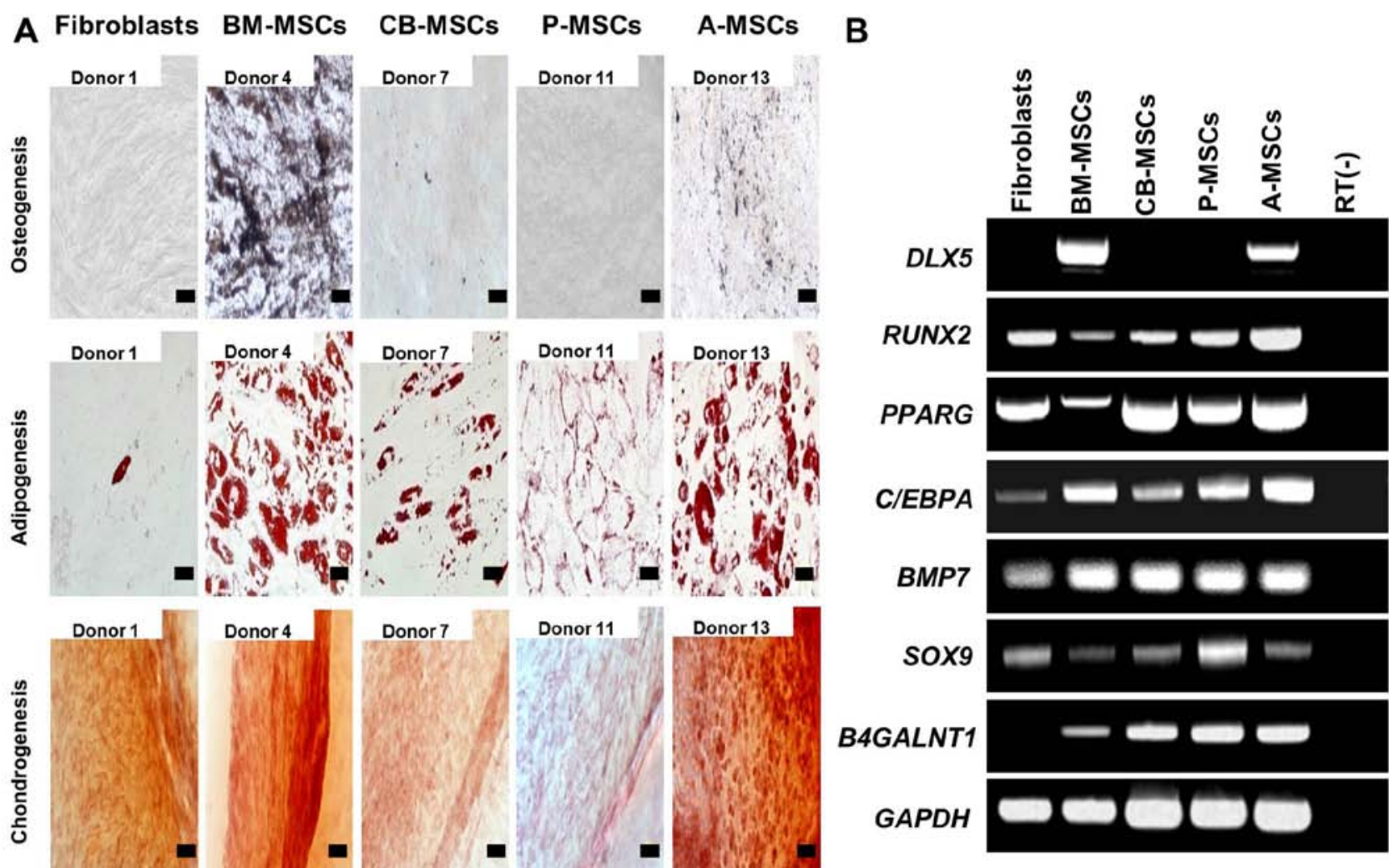

Figure 2. Tri-lineage differentiation of mesenchymal stem cells (MSCs) derived from different tissues. (A) In vitro differentiation assay. MSCs were induced to differentiate toward osteogenic lineage and verified by von Kossa staining after induction (magnification, x200; scale bar, $100 \mu \mathrm{m}$ ), adipogenic lineage and verified by Oil Red O (magnification, x400; scale bar, $50 \mu \mathrm{m}$ ), and chondrogenic lineage and verified by Safranin O staining (magnification, x200; scale bar, $100 \mu \mathrm{m}$ ). One representative of 3 independent experiments is shown. (B) RT-PCR analysis for tri-lineage differentiation-associated markers in MSCs derived from bone marrow (BM-MSCs), umbilical cord blood (CB-MSCs), the placenta (P-MSCs) and adipose tissue (A-MSCs) compared to fibroblasts. The expression of osteogenic (DLX5 and RUNX2), adipogenic (PPARG and C/EBPA) and chondrogenic-associated genes (BMP7 and SOX9) was assayed. The expression of $B 4 G A L N T 1$ was confined to MSCs, and was not noted in fibroblasts. One representative of 3 independent experiments is shown.

DLX5 expression and osteogenic potential. To confirm the differential expression of DLX 5 and osteogenic potential, we performed RT-PCR analysis of DLX5 in various MSCs derived from 3 different donors. DLX5 was expressed in all assessed BM-MSCs and A-MSCs (Fig. 4A). However, DLX5 was also detected in 2 out of 3 CB-MSCs (donors 8 and 9) and 1 of 3 P-MSCs (donor 10), indicating the heterogeneity of MSCs between donors and/or preparations. We analyzed the in vitro osteogenic potential of those MSCs tested for DLX5 gene expression (Fig. 4B). Following osteogenic induction, the BM- and A-MSCs from all 3 donors possessed cells with an osteogenic phenotype. By contrast, the DLX5-expressing CB-MSCs developed an osteogenic phenotype, albeit at varying degrees and this coincided with $D L X 5$ expression (donors 8 and 9). Only a weak osteogenic phenotype was observed in one of the DLX5-expressing P-MSCs, and no osteogenic phenotype was induced in the fibroblasts. It is clear that the levels of $D L X 5$ expression do not necessarily correlate with osteogenic potential. The discrepancy in DLX5 expression and the osteogenic potential of A-MSCs may be explained by the differences in the expression of growth factors, growth factor receptors and transcription factors involved in osteogenesis. Our data suggest that $D L X 5$, one of the key transcription factors for osteoblast differentiation, is a predictive marker for the osteogenic potential of MSCs. In addition, we noted great inter-individual variation in the degree of osteogenic potential between the MSCs obtained from different tissues.

Suppression of $T$ cell proliferation by MSCs derived from different tissues. To assess the immunomodulatory effects of MSCs on activated T cells, we performed a BrdU ELISA assay in T cells co-cultured with various MSCs. The proliferation of T cells was suppressed by MSCs derived from different tissues to varying degrees (Fig. 5A). While the fibroblasts and P-MSCs only weakly inhibited the cell proliferation induced by PHA, a clear reduction in cell proliferation was observed in the BMand A-MSCs.

It is well known that the immunomodulatory properties of MSCs are mediated by HLA and soluble cytokines. The expression of $H L A-A$ and $H L A-G$ was readily detectable in all tested cells, implying that the expression level of $H L A-G$ and MHC class I proteins (HLA-A) in MSCs and fibroblasts could not account for the observed inhibition of $\mathrm{T}$ cell proliferation (Fig. 5B). Expression of HLA-DRB4 was negative in all cells. We then analyzed the gene expression profiles of cytokines related to immunomodulation by RT-PCR that included interleukin 10 (ILIO), TGFB1, tumor necrosis factor, alpha-induced protein 6 [(TNFAIP6), tumor TNF-stimulated gene 6 (TSG-6)] and interleukin 6 (IL6) (Fig. 5C). The relative quantification of gene expression from the MSCs was normal- 

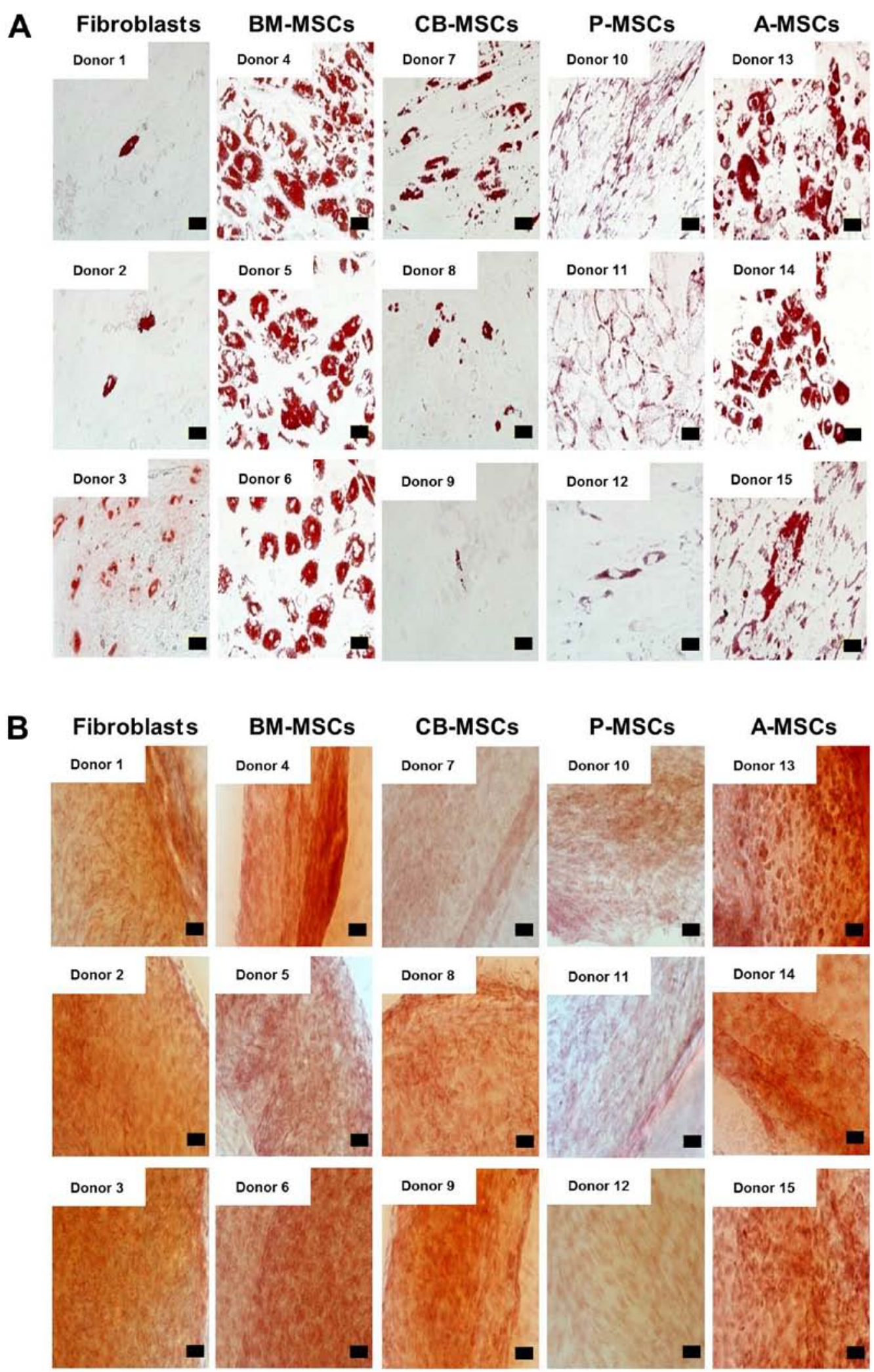

Figure 3. (A) Adipogenenic differentiation potential of mesenchymal stem cells (MSCs) derived from different tissue sources. Adipogenic differentiation was carried out for MSCs and fibroblasts isolated from different donors and terminated after 21 days. Fibroblast, bone marrow (BM)-, cord blood (CB)-, placental (P)-, adipose tissue (A)-derived MSCs from different donors were stained by Oil Red O for intracellular lipid vesicles after induction (x400). (Scale bar, $50 \mu \mathrm{m}$ ). (B) Chondrogenic potential of MSCs derived from different tissue sources. Chondrogenic differentiation was induced for 21 days. Fibroblasts, and bone marrow, cord blood, placental, and adipose tissue-derived MSCs from different donors were induced and analyzed by Safranin-O staining (x200 magnification). (Scale bar, $100 \mu \mathrm{m}$ ).

ized to the internal control, GAPDH. The expression of TGFB1 was higher in the BM-MSCs when compared with the P-MSCs and A-MSCs. Compared to the fibroblasts, no significant differences were detected in the expression of TNFAIP6 and IL6 in 
A
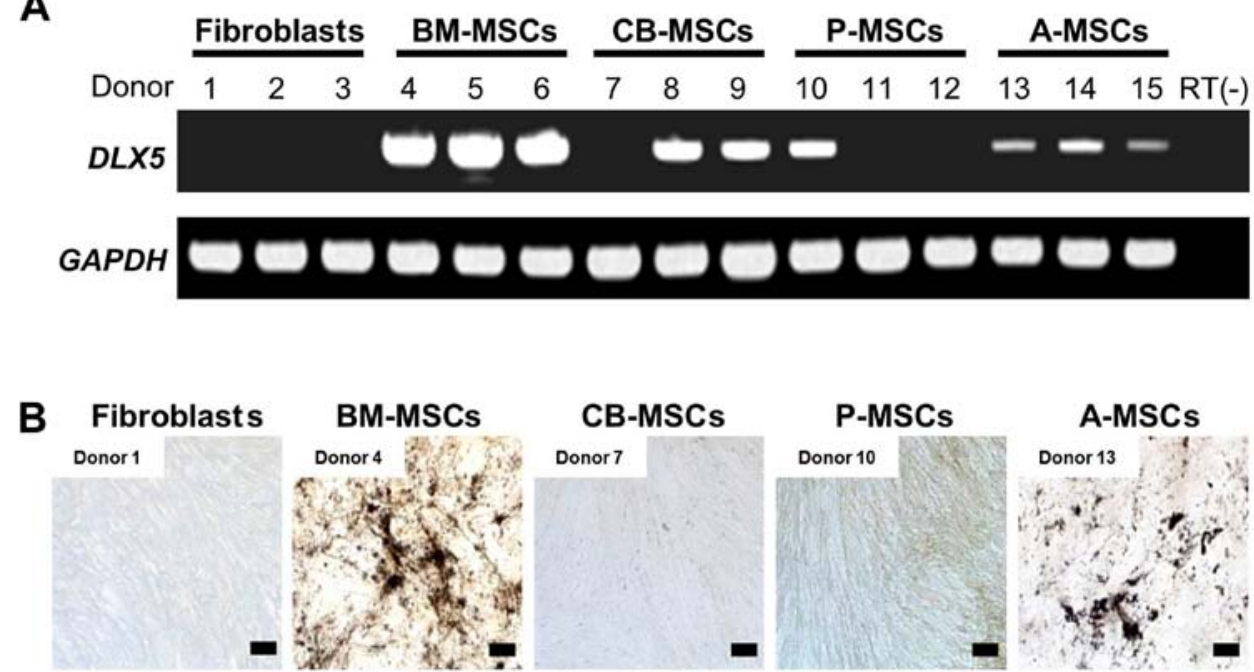

CB-MSCs
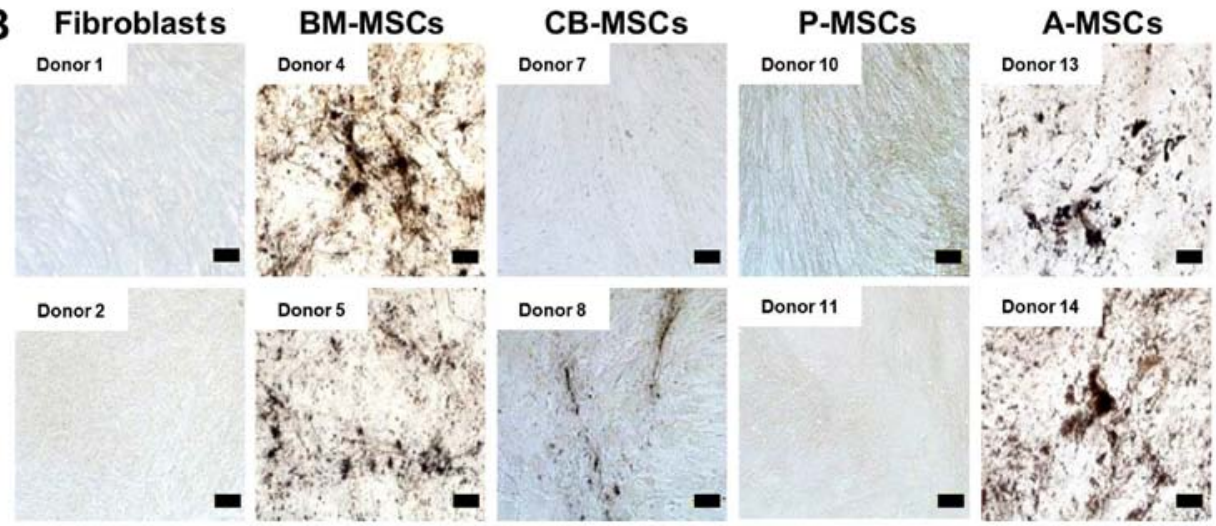

(4) Donor 5

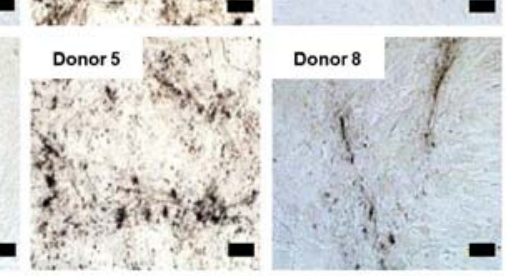

Donor 7

Donor 11

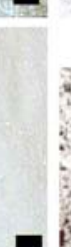

Donor 3
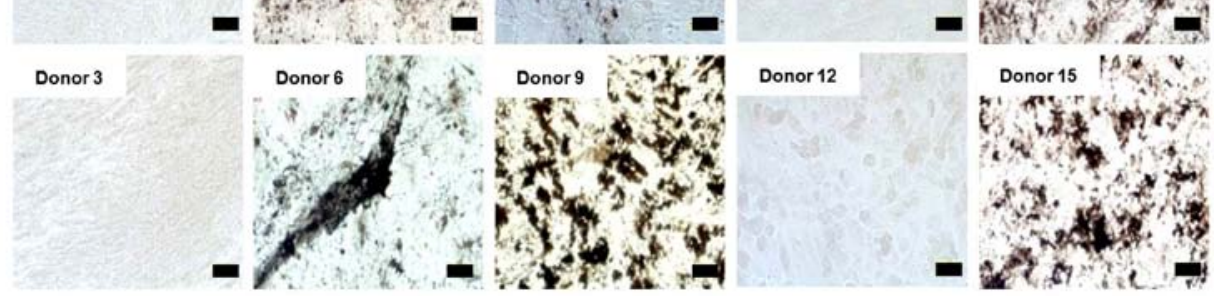

Figure 4. Correlation of $D L X 5$ and osteogenic differentiation capacity of various mesenchymal stem cells (MSCs) from multiple donors. (A) $D L X 5$ transcript of 3 different donors for each MSC derived from different tissues was amplified by RT-PCR. (B) Histologic appearance with von Kossa staining of MSCs of the 3 donors used for RT-PCR in (A). While bone marrow (BM)-derived MSCs and adipose tissue-derived MSCs (A-MSCs) exhibited prominent osteogenic phenotypes, MSCs derived from cord blood and the placenta exhibited inter-donor variation in osteogenic differentiation. (Scale bar, $100 \mu \mathrm{m}$ ).

the MSCs derived from different tissues. Notably, a strong IL10 expression was observed in the BM-MSCs compared to that of fibroblasts and P-MSCs, implying that BM-MSCs exert immunosuppressive activity primarily via $I L I O$.

\section{Discussion}

Due to their regenerative and immunosuppressive properties, MSCs derived from adult tissues have become a preferred cell type in the field of regenerative medicine and are being extensively investigated for their clinical applications (21). Although bone marrow is considered a universal source of multipotent MSCs, the invasive procedure necessary to harvest these cells, the risks of complications and the age-dependent decline of the self-renewal capacity of MSCs has led to a search for alternate sources for MSCs $(22,23)$. CB-MSCs, P-MSCs and A-MSCs have been suggested as alternative sources of MSCs for experimental and clinical purposes since they are free from ethical concerns, easy to procure and are available in large quantities (24-26). Currently, BM-, CB-, P- and A-MSCs are the representative candidates for stem cell therapy (27). As MSCs are being isolated from different tissue sources with different protocols of isolation and culture expansion, it is unclear whether these MSCs share common properties or are dissimilar in terms of certain characteristics that may affect their clinical utilization and outcome. Thus, the comparative analysis of cellular behaviour in vitro, phenotypes, differentiation potential, and immunosuppressive capacity is useful for their potential utilization in clinical settings. In order to characterize MSCs derived from various tissue sources in a number of parameters, all cell preparations in the present study were treated under identical conditions to minimize variables that affect cellular characteristics.

The data obtained demonstrated that MSCs derived from different tissues and the fibroblasts (used as controls) exhibited a similar morphology, clonogenic capacity and immunophenotype, but differed in terms of proliferative rates and differentiation potential. The P-MSCs consistently grew faster and more robustly than the cells derived from other tissues, with a rapid population doubling time. MSCs have a limited life span and enter replicative senescence during in vitro culture, as indicated by enlarged and irregular cell shapes and cessation of proliferation (28). The BM-, CB- and A-MSCs exhibited replicative senescence when they reached passage 10 on average, whereas the P-MSCs expanded until passage 15 . Thus, MSCs are theoretically capable of long-term culture in vitro without losing their fundamental stem cell properties; however, we noted that the growth capacity of the MSCs was 

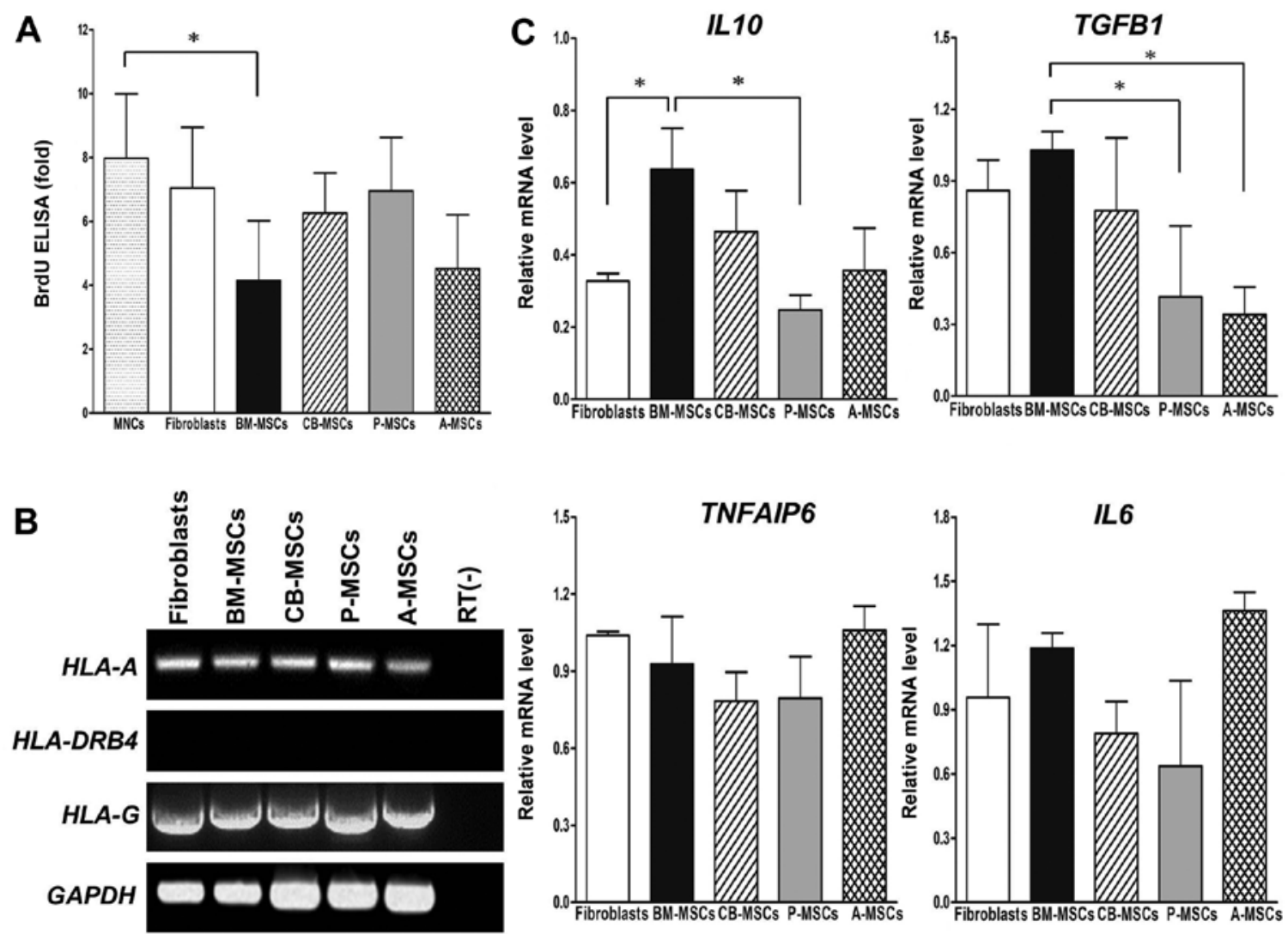

Figure 5. Immunomodulatory effects of mesenchymal stem cells (MSCs) derived from various sources on activated T cells co-cultured with MSCs. (A) Suppression of human peripheral blood mononuclear cells (MNCs) by MSCs. Proliferation of MNCs ( $2 \times 10^{5}$ cells) co-cultured with MSCs (2x10 ${ }^{4}$ cells) from different tissues in the presence of $10 \mu \mathrm{g} / \mathrm{ml}$ of phytohaemagglutinin (PHA) for $72 \mathrm{~h}$ was evaluated by BrdU ELISA. The data represent the means \pm SD of 3 experiments; ${ }^{*}<<0.05$. (B) Gene expression of $H L A-A, H L A-D R B 4$ and $H L A-G$ for immunomodulation in cells derived from various sources. (C) Relative mRNA expression levels of immunosuppressive RT-PCR of interleukin 10 (IL10), transforming growth factor beta 1 (TGFB1), tumor necrosis factor, alpha-induced protein 6 (TNFAIP6) and $I L 6$ in MSCs from different tissues. Expression levels relative to those of the housekeeping gene, GAPDH are shown. The data represent the means \pm SD of 3 experiments; " $\mathrm{p}<0.05$.

generally inferior to that of fibroblasts. Our results demonsrated that P-MSCs are superior to the other MSC types with regard to growth, but more CFU-F colonies were observed among the BM- and CB-MSCs. These results suggest that rapid and longterm growth is not required for the 'stem' properties of MSCs.

Although a list of surface molecules was proposed by ISCT as one of the minimal criteria for MSC identification, all tested markers did not distinguish MSCs from fibroblasts. Thus, the identification of a single definitive marker and precise characterization of MSCs derived from various tissues with regard to their multipotency will be a significant advance for their clinical application. In our phenotypic analysis, we noted that MSCs derived from various sources were positive for the expression of the MSC markers, CD44, CD73, CD90, and CD105, and were negative for CD14, CD34 and CD45. However, CD90 expression, which is known to be associated with haematopoiesis and cell migration, was slightly different among the P-MSCs, and its biological significance needs to be determined. As the function of MSCs is governed by differential molecular profiles, we analyzed the expression of pluripotency genes in order to provide further insight into the differences between MSCs from different tissues. In this study, SOX2, which is involved in self-renewal in pluripotent stem cells and multipotency in MSCs, was only expressed in BM-MSCs, implying the more primitive status of BM-MSCs, as has also been previously noted (29). Since SOX2 functions as a molecular switch in neuronal development, its expression in BM-MSCs may reflect the neuronal differentiation potential (30). BM-MSCs expressed detectable amounts of the majority of core transcription factors, as evidenced by RT-PCR, such as $S O X 2, M Y C, K L F 4$ and $N A N O G$, even in the absence of exogenous stimuli, whereas A-MSCs expressed $M Y C, K L F 4$, NANOG, LIN28 and REXI. The amplified transcripts were of the same size as those in human iPS cells. It was previously demonstrated that INHBA is required for the chondrogenic and osteogenic differentiation of MSCs (31), and our data indicated that the BM- and A-MSCs exhibited a higher expression of $I N H B A$ than the other MSCs. Thus, these data demonstrate that BM- and A-MSCs have properties of primitive multipotent stem cells. KLF4 was ubiquitously expressed in MSCs, as well as fibroblasts.

It is well known that MSCs possess immunosuppressive properties and can inhibit the proliferation and function of major immune cell populations, including $\mathrm{T}$ cells (32). In the present study, in activated T cell co-cultures with MSCs in vitro, only the BM- and A-MSCs significantly inhibited T cell proliferation induced by PHA. While $H L A-G$ expression is known to be involved in the immunomodulation induced by MSCs, we also found that all MSCs and fibroblasts were positive for $H L A-A$ and $H L A-G$, and negative for $H L A-D R B 4$ 
(as shown by RT-PCR), indicating that the expression of HLA molecules is not associated with the inhibitory capacity of PHA-induced T cell proliferation $(33,34)$. However, the possible involvement of $H L A-G$ in the immunosuppression of MSCs via other immune cells cannot be excluded. Other factors associated with the immunomodulatory effects of MSCs include IL10, TGFB1, IL6 and TNFAIP6, TSG-6 $(35,36)$. In the present study, BM-MSCs displayed the greatest suppressive effects on T cells, and elevated levels of ILIO and TGFBI were noted in the BM-MSCs compared to the other MSCs and the fibroblasts, and this is in agreement with the findings of previous studies (37-39).

Concerning the multipotency of MSCs derived from different tissues, their multilineage differentiation capacity was confirmed by in vitro differentiation into osteoblasts, adipocytes and chondrocytes. All of the cells had differentiation potential for at least 2 lineages. In our study, fibroblasts also differentiated toward adipocyte and chondrocyte lineages, as has also been reported previously (20). Only the BM- and A-MSCs differentiated into 3 lineages, including osteoblasts. To identify functional regulator(s) that govern the differentiation potential of MSCs into a specific lineage, we selected 6 genes that are known to play key roles in mesodermal lineage differentiation and verified that only $D L X 5$ is differentially expressed in MSCs with osteogenic potential. Our findings suggest that only BMand A-MSCs have tri-lineage differentiation potential and thus meet the minimal criteria for an MSC, as defined by the ISCT. We also demonstrated that B4GALNT1 (GM2/GS2 synthase), the neural ganglioside GD2 synthase, is expressed by MSCs derived from different tissues. This finding is consistent with the findings of Martinez et al, that GD2 is a valuable marker that uniquely distinguishes MSCs from fibroblasts (40).

DLX5, one of the mammalian homologs of the Drosophila Distal-less $(D L L / D L X)$ genes, is a homeodomain transcription factor that regulates the development of multiple cell types, including osteoblasts and neural cells $(41,42)$. Since $D L X 5$ expression has the potential to identify cells with lineage-specific differentiation capacity, in the present study this was further evaluated in MSCs from multiple donors. In all donors tested, DLX 5 was expressed in MSCs with dominant osteogenic potential, i.e., BM- and A-MSCs. By contrast, 2 of 3 donors of CB-MSC and 1 of 3 donors of P-MSCs expressed $D L X 5$, and the same donors exhibited a concurrent osteogenic phenotype, albeit to varying degrees. Thus, the osteogenic potential of MSCs, regardless of their tissue origin, appears to be related to $D L X 5$ expression. To the best of our knowledge, this is the first study that suggests that $D L X 5$ expression is a predictive maker for MSCs with osteogenic potential. However, it remains to be determined, using a larger number of donors, whether DLX 5 expression firmly characterizes a subset of MSCs with osteogenic potential, although studies of interdonor variation with regard to growth rate, marker expression and multipotency have already been undertaken $(43,44)$.

Our finding of the variation in DLX5 expression between MSCs adds further support to the accumulating evidence that points to substantial diversity both within and between MSCs from various tissue sources (45-47), although little is known regarding the functional differences between MSCs from different tissue and/or different donors. Differences in donor age, gender, genetics, epigenetics and environmental factors have been postulated as the basis for this heterogeneity (48). The issue of MSC heterogeneity has profound implications for clinical application of MSCs, such as establishing standardized protocols that can generate functionally equivalent cellular therapeutics $(49,50)$. Thus, the characterization of MSCs derived from various tissues with standardized protocols will have a great impact on clinical outcomes, such as homing, repairing and/or regenerating damaged tissues.

In conclusion, in this study, we demonstrated that there are significant differences in the characteristics of MSCs derived from various tissue sources and fibroblasts (used as controls), including their multipotency, stemness signature and lineage associated markers. Specifically, the BM- and A-MSCs exhibited full tri-lineage (osteogenic, adipogenic and chondrogenic) differentiation potential, and this ability was associated with the expression of $D L X 5$. In addition, there was a donor-related variation of osteogenic potential in the CB- and P-MSCs, and this potential appeared to be associated with $D L X 5$ expression. In conclusion, the findings of this comparative study contribute to the development of MSC-based cell therapies and regenerative medicine by providing valuable information which can be used when selecting the optimal MSCs for specified clinical applications.

\section{Acknowledgements}

This study was supported by a grant from the Korean Health Technology R\&D Project, Ministry of Health and Welfare, Republic of Korea (HI13C1270).

\section{References}

1. Jiang Y, Jahagirdar BN, Reinhardt RL, Schwartz RE, Keene CD, Ortiz-Gonzalez XR, Reyes M, Lenvik T, Lund T, Blackstad M, et al: Pluripotency of mesenchymal stem cells derived from adult marrow. Nature 418: 41-49, 2002

2. Petersen BE, Bowen WC, Patrene KD, Mars WM, Sullivan AK, Murase N, Boggs SS, Greenberger JS and Goff JP: Bone marrow as a potential source of hepatic oval cells. Science 284: 1168-1170, 1999.

3. Prockop DJ, Gregory CA and Spees JL: One strategy for cell and gene therapy: harnessing the power of adult stem cells to repair tissues. Proc Natl Acad Sci USA 100 (Suppl 1): 11917-11923, 2003.

4. Schwartz RE, Reyes M, Koodie L, Jiang Y, Blackstad M, Lund T, Lenvik T, Johnson S, Hu WS and Verfaillie CM: Multipotent adult progenitor cells from bone marrow differentiate into functional hepatocyte-like cells. J Clin Invest 109: 1291-1302, 2002.

5. Ryan JM, Barry FP, Murphy JM and Mahon BP: Mesenchymal stem cells avoid allogeneic rejection. J Inflamm (Lond) 2: 8, 2005.

6. Mastri M, Lin $\mathrm{H}$ and Lee T: Enhancing the efficacy of mesenchymal stem cell therapy. World J Stem Cells 6: 82-93, 2014.

7. Sharma RR, Pollock K, Hubel A and McKenna D: Mesenchymal stem or stromal cells: a review of clinical applications and manufacturing practices. Transfusion 54: 1418-1437, 2014.

8. Friedenstein AJ, Piatetzky-Shapiro II and Petrakova KV: Osteogenesis in transplants of bone marrow cells. J Embryol Exp Morphol 16: 381-390, 1966.

9. Pittenger MF, Mackay AM, Beck SC, Jaiswal RK, Douglas R, Mosca JD, Moorman MA, Simonetti DW, Craig S and Marshak DR: Multilineage potential of adult human mesenchymal stem cells. Science 284: 143-147, 1999.

10. Bajpai VK, Mistriotis P and Andreadis ST: Clonal multipotency and effect of long-term in vitro expansion on differentiation potential of human hair follicle derived mesenchymal stem cells. Stem Cell Res (Amst) 8: 74-84, 2012.

11. Campagnoli C, Roberts IA, Kumar S, Bennett PR, Bellantuono I and Fisk NM: Identification of mesenchymal stem/progenitor cells in human first-trimester fetal blood, liver, and bone marrow. Blood 98: 2396-2402, 2001 
12. In 't Anker PS, Scherjon SA, Kleijburg-van der Keur C, de Groot-Swings GM, Claas FH, Fibbe WE and Kanhai HH: Isolation of mesenchymal stem cells of fetral or maternal origin from human placenta. Stem Cells 22: 1338-1345, 2004.

13. Ryu KH, Cho KA, Park HS, Kim JY, Woo SY, Jo I, Choi YH, Park YM, Jung SC, Chung SM, et al: Tonsil-derived mesenchymal stromal cells: Evaluation of biologic, immunologic and genetic factors for successful banking. Cytotherapy 14: 1193-1202, 2012.

14. Toma JG, Akhavan M, Fernandes KJ, Barnabé-Heider F, Sadikot A, Kaplan DR and Miller FD: Isolation of multipotent adult stem cells from the dermis of mammalian skin. Nat Cell Biol 3: 778-784, 2001

15. Kern S, Eichler H, Stoeve J, Klüter H and Bieback K: Comparative analysis of mesenchymal stem cells from bone marrow, umbilical cord blood, or adipose tissue. Stem Cells 24: 1294-1301, 2006.

16. Wagner W, Wein F, Seckinger A, Frankhauser M, Wirkner U, Krause U, Blake J, Schwager C, Eckstein V, Ansorge W and Ho AD: Comparative characteristics of mesenchymal stem cells from human bone marrow, adipose tissue, and umbilical cord blood. Exp Hematol 33: 1402-1416, 2005.

17. Dominici M, Le Blanc K, Mueller I, Slaper-Cortenbach I, Marini F, Krause D, Deans R, Keating A, Prockop DJ and Horwitz E: Minimal criteria for defining multipotent mesenchymal stromal cells. The International Society for Cellular Therapy position statement. Cytotherapy 8: 315-317, 2006.

18. Sohn HS, Heo JS, Kim HS, Choi Y and Kim HO: Duration of in vitro storage affects the key stem cell features of human bone marrow-derived mesenchymal stromal cells for clinical transplantation. Cytotherapy 15: 460-466, 2013.

19. Choudhery MS, Khan M, Mahmood R, Mehmood A, Khan SN and Riazuddin S: Bone marrow derived mesenchymal stem cells from aged mice have reduced wound healing, angiogenesis, proliferation and anti-apoptosis capabilities. Cell Biol Int 36: 747-753, 2012

20. Jääger $\mathrm{K}$ and Neuman T: Human dermal fibroblasts exhibit delayed adipogenic differentiation compared with mesenchymal stem cells. Stem Cells Dev 20: 1327-1336, 2011.

21. Ménard $C$ and Tarte $K$ : Immunoregulatory properties of clinical grade mesenchymal stromal cells: evidence, uncertainties, and clinical application. Stem Cell Res Ther 4: 64, 2013.

22. Bianco P, Riminucci M, Gronthos S and Robey PG: Bone marrow stromal stem cells: nature, biology, and potential applications Stem Cells 19: 180-192, 2001.

23. Kemp KC, Hows $\mathrm{J}$ and Donaldson C: Bone marrow-derived mesenchymal stem cells. Leuk Lymphoma 46: 1531-1544, 2005.

24. Bieback K, Kern S, Klüter H and Eichler H: Critical parameters for the isolation of mesenchymal stem cells from umbilical cord blood. Stem Cells 22: 625-634, 2004.

25. Evangelista M, Soncini M and Parolini O: Placenta-derived stem cells: new hope for cell therapy? Cytotechnology 58: 33-42, 2008

26. Ikegame Y, Yamashita K, Hayashi S, Mizuno H, Tawada M, You F, Yamada K, Tanaka Y, Egashira Y, Nakashima S, et al: Comparison of mesenchymal stem cells from adipose tissue and bone marrow for ischemic stroke therapy. Cytotherapy 13: 675-685, 2011.

27. Sousa BR1, Parreira RC, Fonseca EA, Amaya MJ, Tonelli FM, Lacerda SM, Lalwani P, Santos AK, Gomes KN, Ulrich H, et al: Human adult stem cells from diverse origins: an overview from multiparametric immunophenotyping to clinical applications. Cytometry A 85: 43-77, 2014

28. Wagner W, Horn P, Castoldi M, Diehlmann A, Bork S, Saffrich R, Benes V, Blake J, Pfister S, Eckstein V and Ho AD: Replicative senescence of mesenchymal stem cells: a continuous and organized process. PLoS One 3: e2213, 2008.

29. Yoon DS, Kim YH, Jung HS, Paik S and Lee JW: Importance of Sox 2 in maintenance of cell proliferation and multipotency of mesenchymal stem cells in low-density culture. Cell Prolif 44 428-440, 2011.

30. Kishi M, Mizuseki K, Sasai N, Yamazaki H, Shiota K, Nakanishi S and Sasai Y: Requirement of Sox2-mediated signaling for differentiation of early Xenopus neuroectoderm. Development 127: $791-800,2000$
31. Djouad F, Jackson WM, Bobick BE, Janjanin S, Song Y,Huang GT and Tuan RS: Activin A expression regulates multipotency of mesenchymal progenitor cells. Stem Cell Res Ther 1: 11, 2010

32. Shi M, Liu ZW and Wang FS: Immunomodulatory properties and therapeutic application of mesenchymal stem cells. Clin Exp Immunol 164: 1-8, 2011.

33. Nasef A, Mathieu N, Chapel A, Frick J, François S, Mazurier C, Boutarfa A, Bouchet S, Gorin NC, Thierry D and Fouillard L: Immunosuppressive effects of mesenchymal stem cells: involvement of HLA-G. Transplantation 84: 231-237, 2007.

34. Selmani Z, Naji A, Zidi I, Favier B, Gaiffe E, Obert L, Borg C, Saas P, Tiberghien P, Rouas-Freiss N, et al: Human leukocyte antigen-G5 secretion by human mesenchymal stem cells is required to suppress $\mathrm{T}$ lymphocyte and natural killer function and to induce $\mathrm{CD}^{+} \mathrm{CD} 25$ highFOXP ${ }^{+}$regulatory T cells. Stem Cells 26: 212-222, 2008.

35. Bunnell BA, Betancourt AM and Sullivan DE: New concepts on the immune modulation mediated by mesenchymal stem cells. Stem Cell Res Ther 1: 34, 2010

36. Kim HO, Choi SM, Kim HS: Mesenchymal stem cell-derived secretome and microvesicles as a cell-free therapeutics for neurodegenerative disorders. Tissue Eng Reg Med 10: 93-101, 2013.

37. Grütz G: New insights into the molecular mechanism of interleukin-10-mediated immunosuppression. J Leukoc Biol 77: 3-15, 2005.

38. Marie JC, Letterio JJ, Gavin M and Rudensky AY: TGF-beta1 maintains suppressor function and Foxp3 expression in $\mathrm{CD} 4{ }^{+} \mathrm{CD} 25^{+}$regulatory T cells. J Exp Med 201: 1061-1067, 2005.

39. Nasef A, Chapel A, Mazurier C, Bouchet S, Lopez M, Mathieu N, Sensebé L, Zhang Y, Gorin NC, Thierry D and Fouillard L: Identification of IL-10 and TGF-beta transcripts involved in the inhibition of T-lymphocyte proliferation during cell contact with human mesenchymal stem cells. Gene Expr 13: 217-226, 2007.

40. Martinez C, Hofmann TJ, Marino R, Dominici M and Horwitz EM: Human bone marrow mesenchymal stromal cells express the neural ganglioside GD2: a novel surface marker for the identification of MSCs. Blood 109: 4245-4248, 2007.

41. Harada S and Rodan GA: Control of osteoblast function and regulation of bone mass. Nature 423: 349-355, 2003.

42. Long JE, Garel S, Depew MJ, Tobet S and Rubenstein JL: DLX5 regulates development of peripheral and central components of the olfactory system. J Neurosci 23: 568-578, 2003.

43. Phinney DG, Kopen G, Righter W, Webster S, Tremain N and Prockop DJ: Donor variation in the growth properties and osteogenic potential of human marrow stromal cells. J Cell Biochem 75: 424-436, 1999.

44. Portalska KJ, Groen N, Krenning G, Georgi N, Mentink A, Harmsen MC, van Blitterswijk C and de Boer J: The effect of donor variation and senescence on endothelial differentiation of human mesenchymal stromal cells. Tissue Eng Part A 19: 2318-2329, 2013

45. Ho AD, Wagner W and Franke W: Heterogeneity of mesenchymal stromal cell preparations. Cytotherapy 10: 320-330, 2008.

46. Li Z, Zhang C, Weiner LP, Zhang Y and Zhong JF: Molecular characterization of heterogeneous mesenchymal stem cells with single-cell transcriptomes. Biotechnol Adv 31: 312-317, 2013.

47. Sivasubramaniyan K, Lehnen D, Ghazanfari R, Sobiesiak M, Harichandan A, Mortha E, Petkova N, Grimm S, Cerabona F, de Zwart P, et al: Phenotypic and functional heterogeneity of human bone marrow- and amnion-derived MSC subsets. Ann NY Acad Sci 1266: 94-106, 2012.

48. Noer A, Sørensen AL, Boquest AC and Collas P: Stable CpG hypomethylation of adipogenic promoters in freshly isolated, cultured, and differentiated mesenchymal stem cells from adipose tissue. Mol Biol Cell 17: 3543-3556, 2006.

49. Dominici M, Paolucci P, Conte P and Horwitz EM: Heterogeneity of multipotent mesenchymal stromal cells: from stromal cells to stem cells and vice versa. Transplantation 87 (Suppl): S36-S42, 2009.

50. Phinney DG: Functional heterogeneity of mesenchymal stem cells: Implications for cell therapy. J Cell Biochem 113: 2806-2812, 2012. 